\title{
A Discrete 2-D Formulation for 3-D Field Problems With Continuous Symmetry
}

\author{
Bernhard Auchmann ${ }^{1}$, Bernd Flemisch ${ }^{2}$, and Stefan Kurz ${ }^{3}$ \\ ${ }^{1}$ CERN/TE, Geneva 1211, Switzerland \\ ${ }^{2}$ Universität Stuttgart, IWS, Stuttgart 70469, Germany \\ ${ }^{3}$ ETAS GmbH, Stuttgart 70569, Germany
}

In this paper, we describe a general formalism that allows to reduce the spatial dimension of a field problem from 3-D to (2+1)-D. Subsequently, we identify conditions under which the third dimension can be eliminated. We see that the resulting 2-D field problems only decouple if an orthogonality criterion is fulfilled. The approach is based solely on differential-form calculus and can therefore be easily transferred into a discrete setting. As a numerical example, we compute the field of twisted wires.

\section{Index Terms-Continuous symmetries, dimensional reduction, discrete electromagnetism.}

\section{INTRODUCTION}

D IMENSIONAL reduction occurs in different contexts: relativistic 4-D field problems are reduced, mostly implicitly, to 3-D problems with time as a parameter; 3-D problems with symmetries (axi-symmetry, symmetry along a Cartesian axis) are reduced to 2-D problems. This paper constitutes a step in the endeavor to unite all different aspects in a single, differential-form-based framework, and to transfer the equations to a discrete setting. The emphasis is on the application of the theory to a practical numerical problem: the 2-D calculation of the field of twisted wires.

We aim at a dimensional reduction of the curl-curl equation $\mathrm{d} *(1 / \mu) \mathrm{d} A=J$. To this end, we carry out the following steps.

1) Introduce a preferred direction in 3-D space $M$ in the form of a vector field $u$. In our example, the vectors follow helical lines.

2) Fix a 2-D surface $U$ that intersects the flow lines of $u$ by selecting an exact 1-form $\mu=\mathrm{d} \lambda$. The surface is an equipotential of the scalar field $\lambda$. We will set w.l.o.g. $u=\partial_{\lambda}$; see Fig. 2(b).

3) Devise a splitting of the 3-D fields into horizontal and transversal fields (w.r.t. $U$ and $u$ )

$$
\mathrm{S}: \omega \mapsto\left(\begin{array}{c}
\omega_{\|} \\
\omega_{\perp}
\end{array}\right)
$$

For example, using Cartesian coordinates with $u=\partial_{z}$ and $\mu=\mathrm{d} z$, the split vector potential reads $\mathrm{S} A=\left(\begin{array}{c}A_{x} \mathrm{~d} x+A_{y} \mathrm{~d} y \\ A_{z}\end{array}\right)$.

4) Decompose the 3-D operators, e.g., the exterior derivative $\mathrm{d}$ and the Hodge operator $*$, into $2 \times 2$ matrices of operators acting upon the split fields, e.g., $\mathrm{SdS}^{-1}$. We will

Manuscript received December 07, 2009; revised January 29, 2010; accepted February 23, 2010. Current version published July 21, 2010. Corresponding author: B. Auchmann (e-mail: bernhard.auchmann@cern.ch).

Color versions of one or more of the figures in this paper are available online at http://ieeexplore.ieee.org.

Digital Object Identifier 10.1109/TMAG.2010.2045224 end up with a decomposition of the curl-curl equation of the form

$$
\begin{array}{r}
\left(\begin{array}{cc}
-\mathrm{d}_{U} & \partial_{\lambda} \\
0 & \mathrm{~d}_{U}
\end{array}\right) *_{U} \frac{1}{\mu}\left(\begin{array}{cc}
\frac{1}{\eta}\left(1+\eta^{2} \mathrm{j}_{\nu} \mathrm{i}_{v}\right) & -\eta \mathrm{j}_{\nu} \\
-\eta \mathrm{i}_{v} & \eta
\end{array}\right) \\
\quad \times\left(\begin{array}{cc}
\mathrm{d}_{U} & 0 \\
\partial_{\lambda} & -\mathrm{d}_{U}
\end{array}\right)\left(\begin{array}{l}
A_{\|} \\
A_{\perp}
\end{array}\right)=\left(\begin{array}{c}
J_{\perp} \\
J_{\|}
\end{array}\right) .
\end{array}
$$

The quantities $\eta, \nu$, and $v$ will be defined in Section II-B.

5) Discuss the conditions for the above equation system to decompose into two independent equations: continuous symmetry $\left(\partial_{\lambda}=0\right)$ and orthogonality of the flow lines of $u$ and the cutting surface $U(v=\nu=0)$.

6) Discretize the resulting system of 2-D equations, operator by operator, and solve the linear system.

While the focus of this paper is on the reduction from 3-D to (2 +1 )-D, the framework is conceived to deal with arbitrary dimension, and metrics other than the Euclidean metric, e.g., 4-D with Minkowski metric. A more general exposition of the theory can be found in [1]. Section II introduces dimensional reduction in the differential-form framework. Section III proceeds with the discretization of operators, and Section IV presents numerical results. It is assumed that the reader is familiar with differential-form calculus (exterior derivative d, Hodge operator $*$, contraction i, pullback, and push-forward), and the basic concepts of mimetic discretization [2]. A Galerkin approach to helical field problems is found in [3].

\section{DimensionAl REDUCTION}

\section{A. Horizontal and Transversal Fields}

We denote by $\mathcal{X}^{p}(M)$, or just $\mathcal{X}^{p}$, the space of $p$-vector fields of tangent vectors on a 3-D Riemannian manifold $M$, and by $\mathcal{F}^{p}(M)$ the space of differential $p$-forms on $M$. It is useful to define the multiplication operator as

$$
\mathrm{j}: \mathcal{F}^{1} \times \mathcal{F}^{p} \rightarrow \mathcal{F}^{p+1}:(\mu, \omega) \mapsto \mathrm{j}_{\mu} \omega=\mu \wedge \omega .
$$

The duality product $\mathcal{F}^{p} \times \mathcal{X}^{p} \rightarrow \mathcal{F}^{0}$ is denoted by $\cdot \mid \cdot$. The contraction and multiplication operators are dual in the sense 
that $\mathrm{i}_{u} \omega|v=\omega| \mathrm{j}_{u} v$. The operators $\mathrm{i}$ and $\mathrm{j}$ are defined in the obvious way on the respective dual spaces [4].

Let $\mu \in \mathcal{F}^{1}$ be an exact 1-form $\mu=\mathrm{d} \lambda$ that defines a foliation of the 3-D manifold $M . \varphi: U \rightarrow M$ denotes the embedding of a 2-D manifold $U$ in $M$ at $\lambda=$ const. A fibration of $M$ is given by a nonvanishing smooth vector field $u \in \mathcal{X}^{1}$. We require that $\mu \mid u>0$, i.e., the vector field is transversal w.r.t. the foliation. For convenience, we require $\mu \mid u=1$. A differential form is called horizontal if its contraction by the transversal field $u$ yields zero. The space of horizontal forms is denoted

$$
\mathcal{F}_{u}^{p}:=\left\{\omega \in \mathcal{F}^{p} \mid \mathrm{i}_{u} \omega=0\right\} .
$$

Horizontal vectors are tangent to the surfaces $\lambda=$ const. The space of horizontal vectors is defined as

$$
\mathcal{X}_{\mu}^{p}=\left\{v \in \mathcal{X}^{p} \mid \mathrm{i}_{\mu} v=0\right\} .
$$

With the given tools, we now proceed to split a form defined on $M$ into horizontal and transversal components. The horizontal component $\omega_{\|} \in \mathcal{F}_{u}^{p}(M)$ of a form $\omega \in \mathcal{F}^{p}(M)$ is defined by the pullback of $\omega$ to $U$

$$
\omega_{\|}\left|\varphi_{*} v=\varphi^{*} \omega\right| v, \quad v \in \mathcal{X}^{p}(U)
$$

where $\cdot^{*}$ and $\bullet_{*}$ denote the usual pullback and push-forward operators. It can be shown that $\omega_{\|}=\mathrm{i}_{u} \mathrm{j}_{\mu} \omega$. Moreover, each $\omega \in \mathcal{F}^{p}(M)$ can be uniquely represented by a pair of horizontal forms. The operation is called splitting

$$
\begin{aligned}
\mathrm{S}: \mathcal{F}^{p} & \rightarrow \mathcal{F}_{u}^{p} \times \mathcal{F}_{u}^{p-1}: \\
\omega & \mapsto \mathrm{S} \omega=\left(\begin{array}{c}
\mathrm{i}_{u} \mathrm{j}_{\mu} \\
\mathrm{i}_{u}
\end{array}\right) \omega=\left(\begin{array}{c}
\omega_{\|} \\
\omega_{\perp}
\end{array}\right) .
\end{aligned}
$$

The inverse of the splitting is found from $\mathrm{i}_{u} \mathrm{j}_{\mu}+\mathrm{j}_{\mu} \mathrm{i}_{u}=1$

$$
\mathrm{S}^{-1}\left(\begin{array}{c}
\omega_{\|} \\
\omega_{\perp}
\end{array}\right):=\omega_{\|}+\mathrm{j}_{\mu} \omega_{\perp}=\omega .
$$

Although $\mathrm{j}_{\mu} \omega_{\perp}$ represents the transversal component of the form $\omega$, and $\omega_{\perp}$ is a horizontal form, we call $\omega_{\perp}$ the transversal form of $\omega$ as long as there is no risk of ambiguities.

\section{B. Decomposition of Operators}

For the exterior derivative and the Hodge operator, we introduce counterparts (denoted by a hat) that are defined on the horizontal subspaces $\mathcal{F}_{u}^{p}$. These operators arise from the decomposition of operators for a given splitting. The decomposition of the exterior derivative reads

$$
\begin{aligned}
\operatorname{SdS}^{-1} \mathrm{~S} \omega & =\left(\begin{array}{cc}
\mathrm{i}_{u} \mathrm{j}_{\mu} \mathrm{d} & \mathrm{i}_{u} \mathrm{j}_{\mu} \mathrm{dj}_{\mu} \\
\mathrm{i}_{u} \mathrm{~d} & \mathrm{i}_{u} \mathrm{dj}_{\mu}
\end{array}\right)\left(\begin{array}{c}
\mathrm{i}_{u} \mathrm{j}_{\mu} \omega \\
\mathrm{i}_{u} \omega
\end{array}\right) \\
& =\left(\begin{array}{cc}
\hat{\mathrm{d}} & 0 \\
\mathcal{L}_{u} & -\hat{\mathrm{d}}
\end{array}\right)\left(\begin{array}{c}
\omega_{\|} \\
\omega_{\perp}
\end{array}\right)
\end{aligned}
$$

since $\mathrm{j}_{\mu} \mathrm{j}_{\mu}=0$ and $\mathrm{d} \mu=0$. The horizontal exterior derivative is defined as $\hat{\mathrm{d}}:=\mathrm{i}_{u} \mathrm{j}_{\mu} \mathrm{d}$, and $\mathcal{L}_{u}$ denotes the Lie derivative along $u$. It can be shown that $\varphi^{*} \circ \hat{\mathrm{d}}=\mathrm{d}_{U} \circ \varphi^{*}$, with $\mathrm{d}_{U}$ the exterior derivative on the 2-D manifold $U$. Together with $\varphi^{*} \circ \mathcal{L}_{u}=\partial_{\lambda} \circ \varphi^{*}$, we obtain the decomposition used in (2) by applying the pullback of $\varphi$ to (9). For the sake of readability,

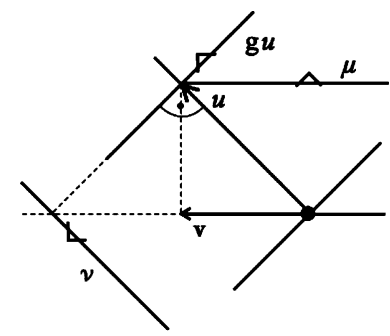

(a)

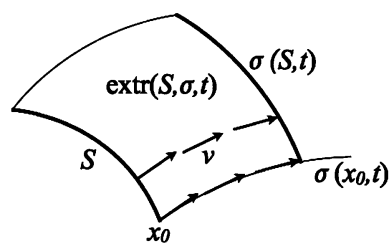

(b)
Fig. 1. (a) Quantities $\nu=(g u)_{\|}$, and $v=\hat{g}^{-1} \nu$ in a tangent space. In this picture, it is assumed that $\|u\|=1$. (b) Extrusion of a 1-manifold $S$ by the flow $\sigma$ of a vector field $v$.

we omit the distinction between horizontal forms and vectors on $M$, and the respective pullback quantities on $U$.

The decomposition of the Hodge operator is more involved. An extensive treatment can be found in [1]. ${ }^{1}$ The decomposition of the Euclidean 3-D Hodge reads

$$
\left(\begin{array}{ll}
0 & 1 \\
1 & 0
\end{array}\right) \mathrm{S} * \mathrm{~S}^{-1}=\hat{*}\left(\begin{array}{cc}
\frac{1}{\eta}\left(1+\eta^{2} \mathrm{j}_{\nu} \mathrm{i}_{v}\right) & -\eta \mathrm{j}_{\nu} \\
-\eta \mathrm{i}_{v} & \eta
\end{array}\right) .
$$

Let $g: \mathcal{X}^{p} \rightarrow \mathcal{F}^{p}$ denote the Riesz isomorphism of the metric on $M$. We define $\hat{g}: \mathcal{X}_{\mu}^{p} \rightarrow \mathcal{F}_{u}^{p}$ such that $\hat{g} v|w=g v| w \forall v, w \in$ $\mathcal{X}_{\mu}^{p}$. If $g_{U}$ denotes the Riesz isomorphism of the induced metric on $U$, then $g_{U}=\varphi^{*} \circ \hat{g} \circ \varphi_{*}$. We can now define all objects in the decomposition (10) by $\eta=\|\mu\|, \nu=(g u)_{\|}$, and $v=$ $\hat{g}^{-1} \nu$; compare Fig. 1(a). The horizontal Hodge is defined as $\hat{*}:=(1 / \eta) \mathrm{i}_{\mu} \mathrm{j}_{\mu} * \mathrm{j}_{\mu}$ and fulfills $\varphi^{*} \circ \hat{*}=*_{U} \circ \varphi^{*}$, which yields the decomposition used in (2).

Note that with $\hat{*}: \mathcal{F}_{u}^{p} \rightarrow \mathcal{F}_{u}^{2-p}$ this decomposition maps $\mathcal{F}_{u}^{p} \times \mathcal{F}_{u}^{p-1} \rightarrow \mathcal{F}^{2-p} \times \mathcal{F}_{u}^{2-(p-1)}$. Consequently, $p$-forms representing Ampère-Maxwell fields are split into columns of ( $p$ 1 )- and $p$-forms, whereas Faraday fields are split into columns of $p$ - and $(p-1)$-forms. A justification for this choice is given in Section III.

\section{Continuous Symmetry and Orthogonality}

To derive the 2-D curl-curl equations of axi-symmetric field problems by dimensional reduction, we use cylindrical coordinates $(r, \varphi, z)$ and set $\mu=\mathrm{d} \varphi, u=\partial_{\varphi}, \eta=(1 / r)$. For axi-symmetry, $\mathcal{L}_{\partial_{\varphi}}=0$, we find with $\nu=0, v=0$

$$
-\mathrm{d}_{U} \frac{1}{r} \frac{1}{\mu} *_{U} \mathrm{~d}_{U} A_{\perp}=J_{\|}, \quad-\mathrm{d}_{U} r \frac{1}{\mu} *_{U} \mathrm{~d}_{U} A_{\|}=J_{\perp} .
$$

Equation (2) decouples into two orthogonal field problems.

For the computation of the field of twisted wires, we use helical coordinates $(R, \phi, Z), R=r, Z=z$, and $\phi=\varphi-\alpha z$. The wires follow flow lines of $u=\partial_{Z}=\alpha \partial_{\varphi}+\partial_{z}$. The foliation is given by $\mu=\mathrm{d} Z=\mathrm{d} z$. We compute $\eta=1, \nu=\alpha R^{2} \mathrm{~d} \phi$, and $v=\alpha \partial_{\phi}$. Despite the symmetry of the field problem $\mathcal{L}_{\partial_{Z}}=0$, the equation system (2) does not decouple into two independent systems. The reason for this lies in the nonvanishing quantities $v$ and $\nu$, which are measures of the nonorthogonality of the flow lines of $u$ with respect to the foliation. In other words, for $g u \wedge \mu \neq 0$, the 2-D equations are not orthogonal and hence coupled.

\footnotetext{
${ }^{1}$ Note that the parameters $v$ and $\nu$ are defined differently. $v$ has to be replaced by $-\xi \eta^{2} v$, and $\nu$ by $-(1 / \xi) \nu$ to reproduce the results in [1].
} 


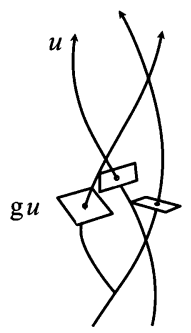

(a)

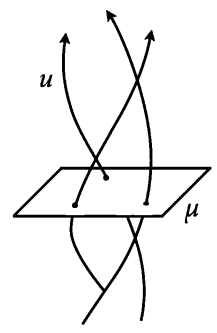

(b)
Fig. 2. Three flow lines of a helical vector field $u$. (a) Orthogonal, nonintegrable decomposition of the local tangent spaces. The spaces do not match to form a submanifold. (b) Integral surface of the closed-form $\mu=\mathrm{d} \lambda$ that is not orthogonal to the flow lines of $u$.

This behavior is not caused by an unfortunate choice of $\mu$. It is a property of the flow lines of $u$. To be orthogonal we had to choose $\mu^{\prime}=f g u$ with some scalar function $f$. However, for $\mu^{\prime}$ to define a foliation of $M$, it has to fulfill the Frobenius integrability condition $\mu^{\prime} \wedge \mathrm{d} \mu^{\prime}=0$. In our case [Fig. 2(a)], we find with $g u=\alpha R^{2} \mathrm{~d} \phi+\left(1+\alpha^{2} R^{2}\right) \mathrm{d} Z$ that any orthogonal choice of $\mu^{\prime}$ is not integrable, whereas integrable choices are not orthogonal. We have to solve the coupled system (2) of two 2-D equations. This finding gives an answer to "open question 7" in [5] whether it is always possible to decouple the horizontal and transversal problems.

\section{DiSCRETIZATION-DisCRETE CONTRACTION}

A mimetic discretization of (2) requires a discrete counterpart for each of the operators. For this paper, we restrict ourselves to 2-D triangular meshes and a first-order approximation of $p$-forms by $p$-cochains and their interpolation by Whitney forms. The derivative operators are well known to be discretized by incidence matrices. For a discrete Hodge operator, we refer to [6], among several possibilities. The 1 -form $\nu$ and the righthand side forms are discretized by the de Rham map. The current 2-cochain is mapped to the dual grid by means of the pairing matrix [7]. The multiplication operator can be readily derived from the discrete cap product [8] that approximates the continuous wedge product.

A discrete contraction operator has been proposed in [9]. We give a brief outline of the concept. The continuous contraction fulfills

$$
\mathrm{i}_{v} \beta\left|S=\lim _{t \rightarrow 0} t^{-1} \beta\right| \operatorname{extr}(S, \sigma, t)
$$

for a 2 -form $\beta$, a 1-D submanifold $S$, and the flow $\sigma$ of a vector field $v .^{2}$ As in [9], we use duality-product notation for the integral of differential $p$-forms over $p$-manifolds. In particular, we are interested in the integral of a contracted Whitney 2-form over an edge of the mesh, as this would, by a de Rham map, correspond to a coefficient of the 1-cochain representative of the contracted 2-cochain; compare (18).

The flow $\sigma: M \times \mathbb{R} \rightarrow M$ of a vector field $v \in \mathcal{X}^{1}(M)$ is defined such that $\sigma(x, t)$ is the flow line starting at $x$ and parameterized by $t$. We have $\sigma(x, 0)=x$ and $\partial_{t} \sigma(x, t)=$ $v(\sigma(x, t))$, i.e., $v$ is tangent in every point to its flow lines. The extrusion in (12) is the union of all points in $S$ and their flow between parameters 0 and $t$; see Fig. 1(b).

\footnotetext{
${ }^{2}$ This is essentially the same as requiring $\mathrm{i}_{v} \beta|w=\beta| \mathrm{j}_{v} w$.
}

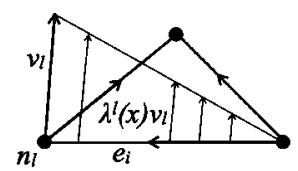

(a)

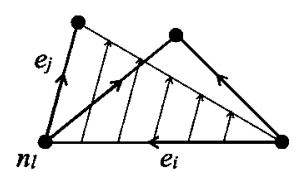

(b)
Fig. 3. (a) Extrusion of an edge $i$ by the flow generated by vector $v^{l}$ in (13). (b) Extrusion of edge $i$ by a flow generated by edge $j$ in (17).

It is suggested in [9] to use a first-order approximation $\sigma(x, t)=x+t v$ of the flow, as higher orders are not relevant for the limit result in (12). This amounts to replacing the flow line by an (affine) geodesic, which is uniquely determined by a point $x$ and a tangent vector $v \in T_{x} M$. Moreover, the vector field is discretized by a de Rham map as a vector-valued 0-cochain, e.g., $v_{l}=v\left(n_{l}\right)$ is the evaluation of $v$ in node number 1 . The node-based approximation of $\sigma$ reads

$$
\sigma_{n}: M \times \mathbb{R} \rightarrow M:(x, t) \mapsto \sum_{l} \lambda^{l}(x)\left(n_{l}+t v_{l}\right)
$$

where $\lambda^{l}$ is the Whitney 0 -form corresponding to node $l$. For the $i$ th coefficient of a contracted 2-cochain, we therefore obtain from (12)

$$
\mathrm{i}_{v} \beta\left|e_{i}=\lim _{t \rightarrow 0} t^{-1} \beta\right| \operatorname{extr}\left(e_{i}, \sigma_{n}, t\right)
$$

where $\beta$ is the Whitney interpolant of the cochain. One term in the flow approximation (13) is depicted in Fig. 3(a). Since Whitney 2-forms are piecewise constant in 2-D, the integral can be easily evaluated. It is linear in $t$, rendering the limit operation trivial.

Further reading in [9] suggests to approximate the flow line in a point $x$ as a 1 -chain. The 1 -chain of a flow line originating in $x$ reads

$$
\operatorname{extr}\left(x, \sigma_{e}, t\right)=\sum_{j} \sum_{l} \lambda^{l}(x) \frac{t}{t_{l}} v_{j}^{l} e_{j}
$$

with

and hence

$$
v_{j}^{l}=w^{j} \mid\left(n_{l}+t v_{l}\right), \quad t \in\left[0, t_{l}\right]
$$

$$
\sigma_{e}:(x, t) \mapsto \sum_{j} \sum_{l} \lambda^{l}(x)\left(n_{l}+\frac{t}{t_{l}} v_{j}^{l} e_{j}\right) .
$$

The coefficient $v_{j}^{l}$ is determined by the integral of the $j$ th Whitney 1 -form $w^{j}$ over the node-based flow line in node $l$ up to parameter $t_{l}$. For computational ease, the parameter $t_{l}$ is chosen such that the flow line is fully contained in a single face or a single edge of the mesh. Equation (17) defines an edge-based approximation of $\sigma$. Even though this looks more complicated than (13), it allows for a straightforward implementation of the operator

$$
\begin{aligned}
\mathrm{i}_{v} \beta \mid e_{i} & =\lim _{t \rightarrow 0} t^{-1} \beta \mid \operatorname{extr}\left(e_{i}, \sigma_{e}, t\right) \\
& =\sum_{j} \sum_{k} \sum_{l} \frac{\frac{1}{2}\left\|e_{i} \wedge e_{j}\right\|}{\left\|f_{k}\right\|} \frac{1}{t_{l}} v_{j}^{l} s^{i j k} \beta_{k}
\end{aligned}
$$

where we use the Whitney interpolation of the 2 -cochain $\beta$ on the face $f_{k}$ which is given by $\left\|f_{k}\right\|^{-1} \beta_{k}$. The factor $s^{i j k}$ equals the orientation or $\left(e_{i} \wedge e_{j}\right)$, if the triangle formed by $e_{i}$ and $e_{j}$ has a nonzero intersection with the positively oriented face $f_{k}$, and 0 otherwise. The norm $\left\|e_{i} \wedge e_{j}\right\|$ is nonzero if the edges $e_{i}$ and $e_{j}$ intersect in one common node. From (18), we can read the coefficients of a discrete contraction matrix that links 


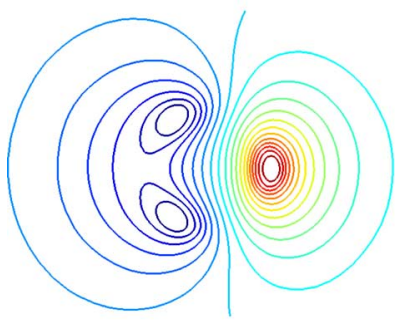

(a)

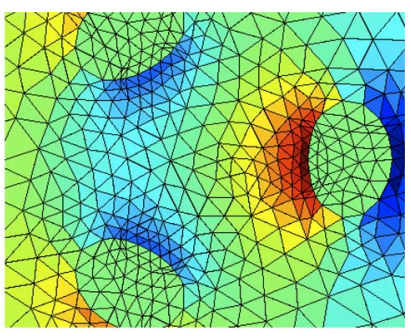

(b)
Fig. 4. Numerical solution $B_{h}$ obtained from the discretization of (2). Field not displayed inside the conductors. (a) Field lines of the transversal 1-cochain $\left(B_{h}\right)_{\perp}$. (b) Detail of the numerical solution; intensity of the horizontal 2 -cochain $\left(B_{h}\right)_{\|}$.

2-cochain coefficients $\beta_{k}$ with the 1-cochain coefficients that result from integrating the contracted form over edges. Equation (18) has been validated by numerical experiments. It differs from [9, eq. (19)] which did not pass validation, as confirmed by [10]. The above concept can be extended to other degrees of cochains and problem dimensions. Formulas that are straightforward for implementation need to be derived. It is to be noted that the edge-based procedure breaks down at the mesh boundary, thus introducing numerical errors. A clear advantage of the use of (17) over (13) or vice versa has not been identified. More work on the subject is found in [11].

With the discrete contraction at hand, we proceed to a mimetic discretization of (2). The kernel of the system matrix is composed from the image of the discrete gradient and the space of constant 0 -cochains. A regularization scheme $S^{\prime}=S+K K^{T}$ is employed, where $S$ is the system matrix, $K$ is a basis of the kernel of $S$, and $S^{\prime}$ is the regularized matrix. This approach is feasible despite the asymmetry of $S$ since $\operatorname{kern} S=\operatorname{kern} S^{T}$. It is in order to ensure this property that we chose to redefine the splitting on the left-hand side of the Hodge operator as $\left(\begin{array}{ll}0 & 1 \\ 1 & 0\end{array}\right) \mathrm{S}=\left(\begin{array}{c}\mathrm{i}_{u} \\ \mathrm{i}_{u} \mathrm{j}_{\mu}\end{array}\right)$ in (10) and (2).

\section{NuMERICAL EXAMPLE}

As an example, we calculate the field of three twisted wires for vanishing sum of the three currents. The computational plane $U$ is given by the plane $z=0$; see Fig. 2(b). Each of the three wires has a diameter of $4 \mathrm{~mm}$ and its center line describes a helix around the $z$-axis of radius $7 \mathrm{~mm}$ and pitch of $44 \mathrm{~mm}$, corresponding to an inclination of about $45^{\circ}$. A formula to compute the intersection of a round twisted wire with the plane $U$ can be found in [12]; see Fig. 4(b).

For error analysis, we choose a circular disc of radius $30 \mathrm{~mm}$ around the $z$-axis. We perform a convergence study starting from a grid consisting of 398 elements, and refining this grid three times up to 25472 elements. We calculate an analytical reference solution on the finest mesh. In particular, we approximate the current density by line-current filaments. Every triangle in the conductors' mesh contains one line current in its barycenter. The current in the filament is proportional to the element size. Ten twist pitches are modeled by several hundred straight line current segments. The reference solution is calculated semianalytically from the set of straight line currents by Biot-Savart's law.

We compare two approximations of the solution. First, we split the semianalytical solution in horizontal and vertical parts,
TABLE I

$L^{2}$ Measures of Errors. $B$ Denotes the Reference, $B_{\mathrm{dR}}$ THE $d R$-MaP APPROXIMATION, AND $B_{h}$ THE NUMERICAL SOLUTION. Fac. IS THE RATIO OF ERRORS OF CONSECUTIVE REFINEMENTS

\begin{tabular}{l|c|c|c|c} 
\#tria. & $\left\|B-B_{\mathrm{dR}}\right\| /\|B\|$ & fac. & $\left\|B-B_{h}\right\| /\|B\|$ & fac. \\
\hline 398 & $2.388 \mathrm{e}-1$ & - & $2.415 \mathrm{e}-1$ & - \\
1592 & $1.238 \mathrm{e}-1$ & 1.93 & $1.234 \mathrm{e}-1$ & 1.96 \\
6368 & $6.125 \mathrm{e}-2$ & 2.02 & $6.222 \mathrm{e}-2$ & 1.98 \\
25472 & $2.670 \mathrm{e}-2$ & 2.29 & $3.058 \mathrm{e}-2$ & 2.03
\end{tabular}

and map them to cochains on the particular mesh by a de Rham (dR) map. We will call this projection the dR-map approximation. Second, the boundary coefficients of the dR-map approximation are used as boundary values for the solution of the discrete 2-D helical field problem that we described above. We call its solution the numerical solution. The transversal field lines and the horizontal field intensity of the numerical solution are depicted in Fig. 4. For both the dR-map approximation and the numerical solution, we eventually use Whitney interpolation and the inverse split (8) to reconstitute the 3-D magnetic fields $B_{\mathrm{dR}}$ and $B_{h}$. Table IV gives the relative error of both w.r.t. the semianalytical solution. The convergence behavior is close to linear with element size; linearity is indicated by a convergence factor of 2 in . $B_{h}$ is close to the optimal projection $B_{\mathrm{dR}}$.

\section{CONCLUSION}

We have derived a (2+1)-D formulation of 3-D magnetostatic field problems. Continuous symmetry along a transversal direction eliminates the third dimension. In case there exists a foliation that is orthogonal to the symmetry direction, the equations can be decoupled, resulting in two independent 2-D field problems. The theory is based solely on differential-form calculus. Hence, it can be discretized operator by operator. We have applied the theory to a field problem with helical symmetry and checked convergence. In the process, we reopened the chapter on the discretization of the contraction operator.

\section{REFERENCES}

[1] S. Kurz, B. Auchmann, and B. Flemisch, "Dimensional reduction of field problems in a differential-forms framework," COMPEL, 2009, accepted for publication.

[2] G. A. Deschamps, "Electromagnetics and differential forms," Proc. IEEE, vol. 69, no. 6, pp. 676-696, Jun. 1981.

[3] A. Nicolet and F. Zolla, "Finite element analysis of helicoidal waveguides," IET Sci. Meas. Technol., vol. 1, no. 1, pp. 67-70, Jan. 2007.

[4] W. H. Greub, Multilinear Algebra. Berlin, Germany: SpringerVerlag, 1967.

[5] P. Raumonen, "Mathematical structures for dimensional reduction and equivalence classification of electromagnetic boundary value problems," Ph.D. dissertation, Tampere Univ. Technol., Tampere, Finland, 2009.

[6] B. Auchmann and S. Kurz, "A geometrically defined discrete Hodge operator on simplicial cells," IEEE Trans. Magn., vol. 42, no. 4, pp. 643-646, Apr. 2006.

[7] B. Auchmann and S. Kurz, "The pairing matrix in discrete electromagnetism," EPJ-Appl. Phys., vol. 39, no. 133, 2007.

[8] S. O. Wilson, "On the algebra and geometry of a manifold's chains and cochains," Ph.D. dissertation, Stony Brook Univ., Stony Brook, NY, Aug. 2005.

[9] A. Bossavit, "Extrusion, contraction: Their discretization via Whitney forms," COMPEL, vol. 22, no. 3, pp. 470-480, 2003.

[10] A. Bossavit, Private Communication. 2009.

[11] H. Heumann and R. Hiptmair, "Extrusion contraction upwind schemes for convection-diffusion problems," ETH Zurich, Zurich, Switzerland, Res. Rep. 2008-30, Oct. 2008.

[12] M. Hardy, "Geometric transformation for double helicalwire rods," M.S. thesis, Univ. Hawaii, Honolulu, HI, 2004. 